\title{
Effect of combined bifidobacteria supplementation and resistance training on cognitive function, body composition and bowel habits of healthy elderly subjects
}

\author{
T. Inoue ${ }^{1}$, Y. Kobayashi ${ }^{2}$, N. Mori ${ }^{1}$, M. Sakagawa ${ }^{1}$, J.-Z. Xiao ${ }^{2}$, T. Moritani ${ }^{3}$, N. Sakane ${ }^{4}$ and N. Nagai ${ }^{1 *}$ \\ ${ }^{1}$ School of Human Science and Environment, University of Hyogo, Hyogo 6700092, Japan; ${ }^{2}$ Next Generation Science Institute, \\ Morinaga Milk Industry Co., Ltd., Kanagawa 2528583, Japan; ${ }^{3}$ Kyoto Sangyo University, Kyoto 6038555, Japan; ${ }^{4}$ Division \\ of Preventive Medicine, Clinical Research Institute for Endocrine and Metabolic Disease, National Hospital Organization, \\ Kyoto Medical Center, Kyoto 6128555, Japan; nagai@shse.u-hyogo.ac.jp
}

Received: 11 December 2017 / Accepted: 9 July 2018

(c) 2018 Wageningen Academic Publishers

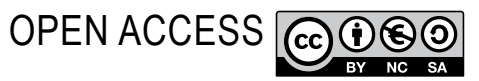

RESEARCH ARTICLE

\begin{abstract}
Physical exercise exerts favourable effects on brain health and quality of life of the elderly; some of these positive health effects are induced by the modulation of microbiota composition. We therefore conducted a randomised, double blind, placebo-controlled trial that assessed whether a combination of Bifidobacterium spp. supplementation and moderate resistance training improved the cognitive function and other health-related parameters in healthy elderly subjects. Over a 12-week period, 38 participants (66-78 years) underwent resistance training and were assigned to the probiotic Bifidobacterium supplementation $\left(\mathrm{n}=20 ; 1.25 \times 10^{10} \mathrm{cfu}\right.$ each of Bifidobacterium longum subsp. longum BB536, B. longum subsp. infantis M-63, Bifidobacterium breve M-16V and B. breve B-3) or the placebo $(\mathrm{n}=18)$ group. At baseline and at 12 weeks, we assessed the cognitive function, using the Japanese version of the Montreal Cognitive Assessment instrument (MoCA-J); modified flanker task scores; depression-anxiety scores; body composition; and bowel habits. At 12 weeks, the MoCA-J scores showed a significant increase in both the groups, while the flanker task scores of the probiotic group increased more significantly than those of the placebo group $(0.35 \pm 0.9$ vs $-0.29 \pm 1.1, P=0.056)$. Only the probiotic group showed a significant decrease in the depression-anxiety scores (5.2 \pm 6.3 to $3.4 \pm 5.5, P=0.012)$ and body mass index ( $24.0 \pm 2.8$ to $23.5 \pm 2.8 \mathrm{~kg} / \mathrm{m}^{2}, P<0.001$ ), with a significant increase in the defecation frequency ( $5.3 \pm 2.3$ to $6.4 \pm 2.3$ times $/ 5$ days, $P=0.023)$ at 12 weeks. Thus, in healthy elderly subjects, combined probiotic bifidobacteria supplementation and moderate resistance training may improve the mental condition, body weight and bowel movement frequency.
\end{abstract}

Keywords: probiotics, resistance training, brain health, bowel habits, body composition

\section{Introduction}

Recent studies have demonstrated a considerable rise in the global population of the elderly. This phenomenon is particularly evident in Japan, a country with one of the highest proportions of elderly citizens in the world (UN, 2015). This demographic change has important implications with respect to disease prevention and healthcare provision. Cognitive impairment is a common issue faced by the elderly and is associated with ageing and dementia. The burden of cognitive impairment is particularly on the rise in the ageing populations. In addition, constipation increases with age (Thompson and Heaton, 1980) and negatively affects the health-related quality of life (QOL) (Wald et al., 2007). In this respect, natural food ingredients are attracting an increasing amount of interest as a means of maintaining health and improving the QOL of the elderly.

Probiotics are living microorganisms proven to exert health benefits on the host when consumed in adequate amounts (Gareau et al., 2010). Human trials have shown that adequate consumption of living microorganisms in the form of probiotics provides a wide range of health benefits (Gareau, 2014), including obesity prevention and management (Kondo et al., 2010). From the viewpoint of the brain-gut axis, gut microbiota may contribute to the 
regulation of brain function through the optimisation of intestinal homeostasis (Quigley, 2017).

Bifidobacterium spp. colonise the human gastrointestinal tract and are one of the major components in human microbiota, with reported health benefits, such as improvement of the intestinal microbiota (Yaeshima et al., 2001), anti-obesity (Kondo et al., 2013), immunemodulation (Ruiz et al., 2017) and infection prevention (Wang et al., 2013). It is noteworthy that certain Bifidobacterium strains may influence the central nervous system through the microbiota-gut-brain axis (Sampson and Mazmanian, 2015). Bercik et al. (2011) reported, based on an animal study, that the administration of Bifidobacterium longum subsp. longum (B. longum) ameliorated the anxietylike behaviours induced by dextran sodium sulphate in mice. Further research found that treatment with Bifidobacterium longum subsp. infantis (B. infantis) restored the behavioural deficits and normalised the proinflammatory cytokines in maternal separation rats (Desbonnet et al., 2010). Recently, Kobayashi et al. (2017) reported that a species of Bifidobacterium breve may prevent cognitive impairment in animal models of Alzheimer's disease. In a human study, the consumption of B. longum 1714 was shown to have the ability to affect the hippocampus-dependent memory performance and frontal midline electroencephalographic mobility in healthy volunteers (Allen et al., 2016).

Numerous studies have demonstrated that physical exercise exerts beneficial effects in terms of improved fitness, disease prevention and QOL enhancement. Moreover, there exists scientific evidence in support of the concept that gut microbiota composition is modulated by physical exercise (Cerdá et al., 2016; Ticinesi et al., 2017). Several animal studies have shown that exercise is associated with microbial diversity and an increase in the beneficial gut bacteria populations. Recently, it has been proposed that this change in the gut microbiota could be the underlying cause of improved health (Cerdá et al., 2016).

Research on elderly populations has shown that resistance training improves cognitive (Walsh et al., 2015) or both cognitive and physical functions (Vaughan et al., 2014). Therefore, it is reasonable to hypothesise that a combination of resistance training and probiotic bifidobacteria supplementation may be superior to either method alone in terms of optimising the mental and physical status and the overall QOL. Therefore, the present randomised, double blind, placebo-controlled trial was conducted to investigate whether combined probiotic Bifidobacterium and moderate resistance training affected cognitive function, mental state, body composition and bowel movement (as a parameter of improved intestinal environment) in healthy elderly subjects. The investigation focused on the mixture of Bifidobacterium strains (B. longum BB536, B. breve M-16V,
B. infantis M-63 and B. breve B-3) to explore its potential in maintaining the QOL of elderly.

\section{Materials and methods}

\section{Participants}

Subjects were recruited via announcements to second-year attendees of a weekly stretch training programme for the elderly at a public liberal art school in the Hyogo prefecture, Japan. Of the 41 interested individuals, 14 male and 25 female subjects met the study inclusion criteria and volunteered for study participation. Those aged $>65$ years who had undergone stretch training for the previous 12 months were included. Those who received public health nursing care, had any contraindications to resistance training, or had been diagnosed with dementia by a physician or were undergoing dementia treatment were excluded.

\section{Ethics statement}

Prior to inclusion, all the participants received a detailed explanation of the study and they provided written informed consent. All study procedures were conducted as per the Declaration of Helsinki. The study was approved by the research ethics committee of the School of Human Science and Environment, University of Hyogo (No. 135, March 4, 2016) and was registered at the University Hospital Medical Information Network Center in Japan (UMIN000021749).

\section{Experimental design}

A randomised, double blind, placebo-controlled trial was conducted. To determine the effects of 12-week, moderate resistance training with or without probiotic supplementation, participants were randomly assigned to the probiotic group $(n=20)$ or the placebo group $(n=19)$. Randomisation was performed by an independent organisation (Laboratory of Family Medicine, Jichi Medical University, Japan). Subjects and investigators responsible for assessing the outcomes were blinded to the randomisation status. On completion of data collection, all the data were fixed. Thereafter, statistical analyses were conducted. During the 12-week study period, compliance with the study protocol was assessed at 2-week intervals via collection of unused probiotic supplements, and the inspection of daily records of supplement ingestion and home training (type and duration). All study measurements were performed at baseline and at 12 weeks.

\section{Products administered}

We prepared a sachet containing lyophilised powder of $B$. longum BB536, $B$. infantis M-63, B. breve M-16V and $B$. breve B-3 (approximately $1.25 \times 10^{10} \mathrm{cfu}$ each; Morinaga Milk Industry Co., Ltd., Kanagawa, Japan) with the carrier 
dextrin. Participants were instructed to consume one sachet of the supplement or the placebo with water (preferably after breakfast) every day for 12 weeks.

\section{Nutritional status}

For baseline information, the subjects' nutritional status was evaluated by a registered dietician using (1) the short version of the Mini Nutritional Assessment (MNA) (Lilamand et al., 2015) and (2) a self-reported record of all beverages and weighed food consumed on a typical weekday prior to the baseline interview.

\section{Resistance training programme}

All the participants participated in a 12 -week resistancetraining programme at the public liberal art school. The training classes were supervised by an experienced sports scientist and a Tai Chi master. Each training class comprised the following: a warm-up session $(30 \mathrm{~min})$; resistance training ( $40 \mathrm{~min}$; combination of latex band training [Theraband, Performance Health, Akron, OH, USA], squat and Tai Chi); and a cool-down session (20 min). In addition, participants were encouraged to perform daily exercises at home, such as stretching, squatting, or walking and record the types and duration of each exercise session.

\section{Measurement of cognitive function}

The general cognitive function was measured by a trained research assistant using the Japanese version of the Montreal Cognitive Assessment instrument (MoCA-J) (Fujiwara et al., 2010). The MoCA-J scores range from 0 to 30, with higher scores indicating more optimal cognitive performance. This test includes an assessment for the education level. Executive function and inhibitory controls were measured using a flanker task, a set of response inhibition tests, comprising two types of stimuli, the central target letter of which is flanked by noise letters (Kamijo et al., 2009). To measure the response accuracy and reaction time, we developed a modified flanker task test programme. Using a touch screen tablet (SurfacePro3, Microsoft Japan Ltd., Tokyo, Japan), 24 situations (12 congruent and 12 incongruent) were presented in random order. Five arrowheads were displayed per situation. The middle (target) arrowhead was surrounded by flanker arrowheads. In the congruent situation, all arrowheads pointed in the same direction as the middle arrowhead $(<<<<<$ or $>>>>>)$. In the incongruent condition, the middle arrowhead pointed in the opposite direction to that of the surrounding arrowheads $(<<><<$ or $\rangle>\langle>\rangle)$. Using their index finger, participants were instructed to press an image on the screen as quickly as possible in response to the direction of the middle (target) arrowhead (left $[>]$ or right $[<]$ ) and perform as many tasks as possible within $1 \mathrm{~min}$. The number of correct responses and the average reaction time (s) were used for evaluating task performance.

\section{Depression and anxiety scores}

The mental state was assessed using the Japanese versions of the following brief self-report questionnaires: (1) Patient Health Questionnaire-9 (PHQ-9) (Kroenke et al., 2001; Muramatsu et al., 2007) and (2) Generalised Anxiety Disorder-7 (GAD-7) (Muramatsu et al., 2010; Spitzer et al., 2006). The PHQ-9 comprises nine items (the GAD-7 includes seven items), with four possible ranked responses each (e.g. question: over the past 2 weeks, how often have you been bothered by the following problems? Feeling nervous, etc.; possible responses: not at all [score, 0]; several days [score, 1]; more than half the days [score, 2]; nearly every day [score, 3]) that have been well validated in general populations (Löwe et al., 2008, 2010). The total PHQ-9 scores (from 9 to 27) were used as depression scores and total GAD-7 scores were used as anxiety scores (from 7 to 21), with a higher score indicating higher levels of depression symptomatology or anxiety. Summed PHQ-9 and GAD-7 scores were used as scores of the overall mental state (from 16 to 46 ).

\section{Clinical measurements}

Height and body weight were measured using a stadiometer and digital scale, respectively, according to standardised anthropometric procedures. Body weight was measured to the nearest $0.1 \mathrm{~kg}$ (BC-315, Tanita, Tokyo, Japan) with the participant in light clothing and barefoot. Body mass index (BMI) was calculated. To evaluate the body composition, a bioelectrical impedance analyser (InBody S10, BioSpace, Seoul, Korea) was used. Blood pressure levels were measured using a mercury sphygmomanometer (HEM-7250-IT; OMRON, Co., Ltd., Kyoto, Japan), with the participant in a sitting position. Biochemical analyses were conducted as described previously (Nagai et al., 2011).

\section{Bowel movement characteristics}

From a month prior to the intervention, each participant was asked to self-record defecation frequency, faecal characteristics and defecation sensation on 5 consecutive days before (as baseline assessment) and after the intervention period (as the 12-week assessment). The participant was asked to assess faecal characteristics using the modified Bristol Stool Form Scale (BSFS) (O'Donell et al., 1990). The faecal shape was categorised as follows: (1) pellets; (2) hard; (3) quite hard; (4) normal; (5) semi-solid; (6) mud-like; and (7) watery. Defecation sensation was divided into the following five categories: (1) uncomfortable; (2) slightly uncomfortable; (3) normal; (4) slightly comfortable; (5) comfortable (Kondo et al., 2013). 


\section{Sample size}

Sample size was calculated using the MoCA-J scale as the main outcome. This calculation showed that a sample size of 21 participants per group was necessary to detect an inter-group difference of 2 points on the MoCA-J scale (power $=0.8$, alpha $=0.05$ ) between baseline and 12 weeks (G*Power, version 3.1.9; Faul et al., 2009). This predicted difference equated to an effect size of $\geq 0.8$.

\section{Statistical analyses}

All parameters are expressed as means \pm standard deviation values. All statistical analyses were conducted using the Statistical Package for the Social Sciences (SPSS for Windows ${ }^{\text {mtw }}$ ver. 22, IBM Inc., Tokyo, Japan). Prior to the statistical evaluation, normality testing was conducted using the Kolmogorov-Smirnov test. Baseline differences between the probiotic and placebo groups were tested using unpaired $t$-tests. Intra-group changes in the values between baseline and 12 weeks were tested using paired $t$-tests. To determine whether the measurements were influenced by probiotic supplementation, two-way analysis of variance with repeated measurement was conducted. Here, the factors were group (probiotic or placebo) and time (before and after intervention). Statistical significance was defined as $P<0.05$.

\section{Results}

\section{Participant flow and baseline characteristics}

A Consolidated Standards of Reporting Trials flow diagram of the study is provided in Figure 1. Thirty-nine participants fulfilled the inclusion criteria and were randomly assigned to the probiotic $(n=20)$ or the placebo group $(n=19)$. One female participant in the probiotic group dropped out during the intervention period owing to challenges in complying with the study protocol. Total 38 (97.4\%) participants (probiotics, $n=20,100 \%$; placebo, $n=18,94.7 \%$ ) completed the 12-week intervention study. During the study, no adverse effects were reported following the administration of the probiotics or the placebo. The baseline characteristics of the study subjects are shown in Table 1 . No significant differences were noted between the study groups at baseline.

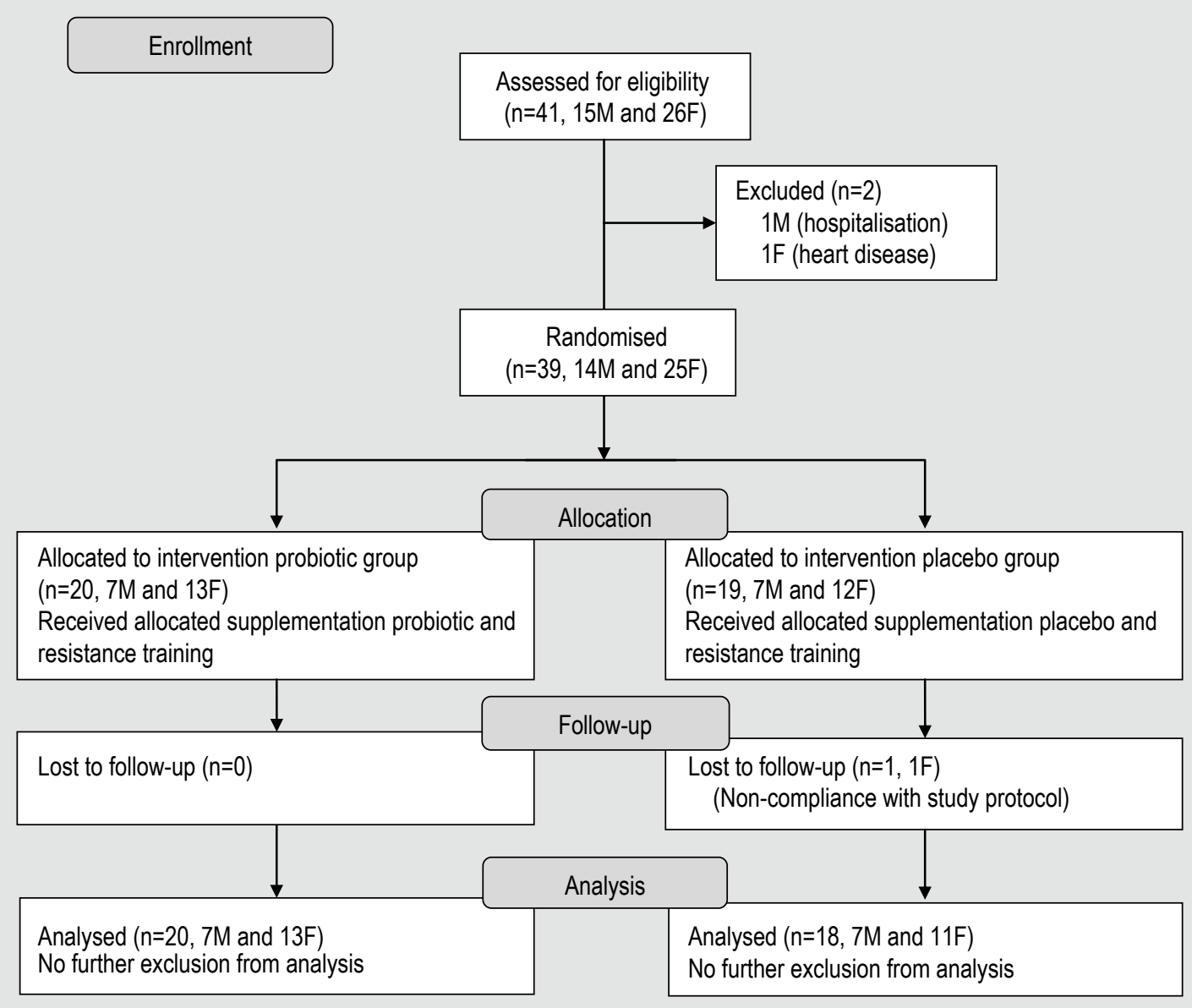

Figure 1. Flow diagram of the study. 
Table 1. Participant characteristics at baseline. ${ }^{1}$

\begin{tabular}{lllll} 
& All participants & Probiotic group & Placebo group & $P_{\text {-value }}{ }^{2}$ \\
Number & & & & \\
Female, number (\%) & 38 & 20 & 18 & 0.31 \\
Age (years) & $24(63.2)$ & $13(65.0)$ & $11(61.1)$ & 0.40 \\
Education (years) & $70.3 \pm 3.1$ & $69.9 \pm 3.0$ & $70.9 \pm 3.2$ & 0.46 \\
Height (cm) & $11.6 \pm 2.1$ & $11.4 \pm 2.3$ & $11.9 \pm 2.0$ & 0.20 \\
Body mass (kg) & $157.5 \pm 7.4$ & $158.4 \pm 7.8$ & $156.6 \pm 6.9$ & 0.29 \\
Body mass index (kg/m ${ }^{2}$ ) & $58.6 \pm 9.9$ & $60.6 \pm 11.5$ & $56.4 \pm 7.3$ & 0.36 \\
Lean body mass (kg) & $23.5 \pm 2.8$ & $24.0 \pm 2.8$ & $23.0 \pm 2.7$ & 0.50 \\
Body fat (\%) & $41.9 \pm 6.8$ & $42.9 \pm 7.5$ & $40.9 \pm 5.9$ & 0.32 \\
Systolic blood pressure (mmHg) & $28.1 \pm 6.4$ & $28.8 \pm 6.0$ & $27.3 \pm 7.0$ & 0.34 \\
Diastolic blood pressure (mmHg) & $136 \pm 18$ & $139 \pm 21$ & $133 \pm 15$ & 0.98 \\
Energy intake (kJ per day) & $74 \pm 10$ & $76 \pm 10$ & $73 \pm 9$ & 0.23 \\
Duration of habitual exercise (min per week) & $8,845 \pm 1,280$ & $8,840 \pm 1,176$ & $8,849 \pm 1,423$ & \\
\hline${ }^{1}$ Values are expressed as mean \pm standard deviation. & $434 \pm 166$ & $403 \pm 173$ & $469 \pm 156$ & \\
${ }^{2}$ Unpaired t-test. & & & & \\
\hline
\end{tabular}

According to the MNA and dietary food records at baseline, no participant was malnourished. Almost all the participants consumed dairy products on a daily basis; however, it is noteworthy that the baseline intake of dairy products was comparable between the groups (probiotics: $185 \pm 118$ g, placebo: $195 \pm 137 \mathrm{~g}, P=0.82$ ). The participants were advised to maintain their dietary habits during the intervention period; therefore, the amount of consumption of dairy products, including yogurt, remained unchanged. Moreover, none of the participants consumed commercial probiotic supplements before and during the study.

\section{Compliance}

During the 12-week intervention period, probiotic and placebo group participants performed similar training in terms of the percentage of resistance training classes attended and the frequency of performing home exercise (probiotics, $83.5 \pm 18.7 \%$; placebo, $90.1 \pm 15.9 \%, P=0.25$ ). In both the groups, compliance for the assigned study supplementation was high (probiotics, $99.8 \pm 0.6 \%$; placebo, $99.3 \pm 1.9 \%, P=0.25)$. The biochemical analyses results showed no significant differences between the groups before or after the intervention (Supplementary Table S1), and there were no adverse events resulting from the probiotic supplementation.

\section{Cognitive function (primary outcome)}

Table 2 shows the parameters of cognitive function in the probiotic and placebo group participants at baseline and at 12 weeks. For the general cognitive function, a significant increase in the MoCA-J scores was observed in both the groups at 12 weeks (probiotics $P<0.001$; placebo, $P<0.01$ ). The group $\times$ time interaction was insignificant $(P=0.54)$, indicating that probiotic Bifidobacterium had no significant impact on the MoCA-J scores. For executive function, no significant change in the number of correct responses or the average reaction time in the flanker task was observed at 12 weeks. The number of changes in terms of the level of correct responses tended to be higher in the probiotic group than in the placebo group (group $\times$ time interaction, $P=0.056)$.

\section{Mental state scores (secondary outcome)}

Table 3 shows the parameters of mental health for the probiotic and placebo groups at baseline and at 12 weeks. Participants in both the groups had low depression, anxiety and total scores at baseline; there was no significant difference in these scores of the two groups (depression: $P=0.84$, anxiety: $P=0.50$, total: $P=0.62$, probiotic vs placebo group), indicating that participants of both the groups were in good general mental health at study inclusion. At 12 weeks, a significant decrease was observed in the anxiety scores of the placebo group $(P<0.05)$, while a significant decrease was observed in the total mental state score of the probiotic group $(P<0.05)$.

\section{Body composition (secondary outcome)}

Table 4 shows the parameters of body composition for the probiotic and placebo groups at baseline and at 12 weeks. At 12 weeks, the probiotic group showed a significant decrease in the body weight, BMI and body fat $(P<0.001$, each). At 12 weeks, the placebo group showed a significant decrease 
Table 2. Cognitive function. ${ }^{1}$

\begin{tabular}{|c|c|c|c|c|c|}
\hline & \multicolumn{2}{|l|}{ Probiotic group $(n=20)$} & \multicolumn{2}{|l|}{ Placebo group ( $n=18$ ) } & \multirow[t]{2}{*}{$P$-value ${ }^{2}$} \\
\hline & Baseline assessment & 12 weeks assessment & Baseline assessment & 12 weeks assessment & \\
\hline \multicolumn{6}{|l|}{ General cognitive function } \\
\hline MoCA-J (score) $)^{3}$ & $23.5 \pm 3.4$ & $25.8 \pm 3.6^{* * *}$ & $23.9 \pm 2.9$ & $25.8 \pm 2.5^{\star *}$ & 0.54 \\
\hline \multicolumn{6}{|l|}{ Flanker task } \\
\hline Correct response (number) & $22.6 \pm 4.9$ & $23.0 \pm 4.5$ & $23.3 \pm 1.8$ & $23.0 \pm 2.8$ & 0.056 \\
\hline Reaction time (s) & $1.81 \pm 0.54$ & $1.96 \pm 1.25$ & $1.74 \pm 0.33$ & $1.88 \pm 0.54$ & 0.95 \\
\hline
\end{tabular}

Table 3. Depression and anxiety scores. ${ }^{1}$

\begin{tabular}{|c|c|c|c|c|c|}
\hline & \multicolumn{2}{|l|}{ Probiotic group $(n=20)$} & \multicolumn{2}{|l|}{ Placebo group (n=18) } & \multirow[t]{2}{*}{$P$-value ${ }^{2}$} \\
\hline & Baseline assessment & 12 weeks assessment & Baseline assessment & 12 weeks assessment & \\
\hline Depression (score, 0-27) & $2.6 \pm 2.7$ & $1.9 \pm 2.6$ & $2.8 \pm 2.7$ & $2.3 \pm 2.3$ & 0.73 \\
\hline Anxiety (score, 0-21) & $2.1 \pm 2.7$ & $1.4 \pm 2.9$ & $1.6 \pm 1.4$ & $0.9 \pm 1.4^{*}$ & 0.85 \\
\hline
\end{tabular}

${ }^{1}$ Values are expressed as mean \pm standard deviation. No significant difference at baseline between the probiotic and placebo groups.

${ }^{*} P<0.05$, within the group from baseline to 12 weeks assessment.

2 Significant difference between the treatment and placebo groups in repeated measurement ANOVA assessing group $\times$ time interaction.

Table 4. Body composition. ${ }^{1}$

\begin{tabular}{|c|c|c|c|c|c|}
\hline & \multicolumn{2}{|l|}{ Probiotic group $(n=20)$} & \multicolumn{2}{|l|}{ Placebo group (n=18) } & \multirow[t]{2}{*}{$P$-value ${ }^{2}$} \\
\hline & Baseline assessment & 12 weeks assessment & Baseline assessment & 12 weeks assessment & \\
\hline Body mass (kg) & $60.6 \pm 11.5$ & $59.4 \pm 11.3^{* * *}$ & $56.4 \pm 7.3$ & $55.8 \pm 7.6$ & 0.11 \\
\hline Body mass index $\left(\mathrm{kg} / \mathrm{m}^{2}\right)$ & $24.0 \pm 2.8$ & $23.5 \pm 2.8^{* \star *}$ & $23.0 \pm 2.7$ & $22.8 \pm 2.8$ & 0.11 \\
\hline Percentage of body fat (\%) & $28.8 \pm 6.0$ & $27.0 \pm 5.6^{\star \star \star}$ & $27.3 \pm 7.0$ & $25.6 \pm 6.5^{\star *}$ & 0.89 \\
\hline Lean body mass $(\mathrm{kg})$ & $42.9 \pm 7.5$ & $43.1 \pm 7.6$ & $40.9 \pm 5.9$ & $41.4 \pm 5.8^{*}$ & 0.37 \\
\hline
\end{tabular}

${ }^{1}$ Values are expressed as mean \pm standard deviation. No significant difference at baseline between the probiotic and placebo groups. ${ }^{*} P<0.05,{ }^{*} P<0.01$, *** $P<0.001$ within the group from baseline to 12 weeks assessment.

2 Significant difference between the probiotic and placebo groups in repeated measurement ANOVA assessing group $\times$ time interaction.

in the body fat $(P<0.01)$ and a significant increase in the lean body mass $(P<0.05)$. The number of changes in the body composition values did not differ between the groups (group $\times$ time interaction, $P>0.05$ ).

\section{Bowel habits (secondary outcome)}

Table 5 shows the parameters of bowel habits, indicators of intestinal environment, for the probiotic and placebo groups at baseline and at 12 weeks. At 12 weeks, there was 
Table 5. Bowel habit characteristics. ${ }^{1}$

\begin{tabular}{|c|c|c|c|c|c|}
\hline & \multicolumn{2}{|l|}{ Probiotic group $(n=20)$} & \multicolumn{2}{|l|}{ Placebo group ( $n=18$ ) } & \multirow[t]{2}{*}{$P$-value ${ }^{2}$} \\
\hline & Baseline assessment & 12 weeks assessment & Baseline assessment & 12 weeks assessment & \\
\hline $\begin{array}{l}\text { Defecation frequency (number } \\
\text { of times in } 5 \text { consecutive days) }\end{array}$ & $5.3 \pm 2.3$ & $6.4 \pm 2.3^{*}$ & $5.8 \pm 2.0$ & $6.1 \pm 2.2$ & 0.36 \\
\hline $\begin{array}{l}\text { Average score for stool form } \\
\left.\text { and consistency }{ }^{3} \text { (score, } 1-7\right)\end{array}$ & $3.6 \pm 0.9$ & $3.6 \pm 0.7$ & $3.2 \pm 0.8$ & $3.7 \pm 0.8$ & 0.16 \\
\hline $\begin{array}{l}\text { Average score for comfort after } \\
\text { defecation } 4 \text { (score, } 1-5)\end{array}$ & $3.4 \pm 1.2$ & $3.4 \pm 1.1$ & $3.1 \pm 1.2$ & $3.0 \pm 0.9$ & 0.84 \\
\hline
\end{tabular}

a significant increase $(P<0.05)$ in the defecation frequency of the probiotic group, with no change in the scores for stool form or defecation sensation. The number of changes in the bowel movement values was similar for the groups (group $\times$ time interaction, $P>0.05$ ).

\section{Discussion}

Increasing evidence suggests that probiotics may optimise physiological functions, such as epithelial barrier function, gut homeostasis and the immune response (Gareau, 2014). In addition, recent research suggests that probiotics may alter the brain function and exert a beneficial effect for psychiatric and neurological diseases (Mu et al., 2016; Quigley, 2017). Research on elderly populations has shown that resistance exercise improves their cognitive function and other health-related parameters (Vaughan et al., 2014; Walsh et al., 2015). The mechanisms through which exercise exerts a beneficial effect on brain health include optimisation of the effects on the hypothalamic-pituitaryadrenal (HPA) axis, minimising the inflammatory state and enhancing nerve growth factor expression that contributes to neural plasticity (Silverman and Deuster, 2014). There is evidence supporting the concept that physical exercise can modulate gut microbiota composition, increasing the diversity and representation of the key taxa with healthpromoting metabolic activities, such as production of shortchain fatty acids (SCFA) (Barton et al., 2017; Matsumoto et al., 2008). SCFA reportedly have various beneficial functions, such as gene regulation, immune regulation, mitochondrial biogenesis and maturation and functioning of the brain microglia, the most abundant resident immune cells in the brain related to brain inflammatory condition (Fung et al., 2017). In addition to SCFA production, commensal microbiota were reported to affect the HPA axis (Sudo et al., 2004) and neuronal plasticity (Diaz Heijtz et al., 2011), both of which could be positively regulated by physical exercise. Therefore, we hypothesised that physical exercise and intestinal microbiota interacted with each other and manifested an additional or a synergistic effect when physical exercise and probiotics were combined.

The present randomised, double blind, placebocontrolled trial investigated whether combined probiotic Bifidobacterium supplementation and moderate resistance training affects cognitive function and other health-related parameters in healthy elderly subjects. Both exercise and probiotics were reported to affect brain activity, body composition and gastrointestinal condition, and these parameters are reported to be closely associated and influenced by each other (Bhatia and Tandon, 2005; Gallucci et al., 2013; Gustafson et al. 2003; Portincasa et al., 2009). Therefore, we chose these parameters in this study. At 12 weeks, the cognitive function, measured using the MoCA-J scores, improved significantly in both the study groups, while the flanker task scores tended to be higher in the probiotic group. In addition, there were significant decreases in the depression-anxiety scores and BMI, with a significant increase in the defecation frequency at 12 weeks in the probiotic group.

Research has demonstrated the existence of bidirectional, autonomic nervous system- and humoral factor-mediated brain-gut axis communication (Al Omran and Aziz, 2014); it has also been shown that intestinal microbiota composition may affect intracerebral nerve factors and neurotransmitters (Mu et al., 2016). Similar to our findings, the report by Chung et al. (2014) showed improvements in several cognitive function parameters in healthy elderly subjects after the ingestion of Lactobacillus helveticus-fermented 
milk for 12 weeks, although no significant differences were found against the placebo group. In contrast to previous reports (Akbari et al., 2016; Chung et al., 2014), we observed a significant increase in the MoCA-J scores of both the groups. This may be attributable to the fact the present cohort received concomitant resistance training. Given that exercise exerts favourable effects on geriatric cognitive function (Northey et al., 2017; Xu et al., 2017), the observed increase in the MoCA-J score in the two study groups may have been due to training effects. In addition, the baseline MoCA-J scores were relatively high. This indicates that the subjects showed good cognitive function; this may limit the scope for improvement (ceiling effect). Future studies involving elderly cohorts with a larger sample size or mild cognitive impairment are warranted to elucidate the impact of Bifidobacterium on cognitive function.

In addition to the improvement in cognitive function, research suggests that probiotics may ameliorate mental disorders, such as depression and anxiety (Cryan and Dinan, 2012). Using a cohort of healthy adults, Messaoudi et al. (2011) conducted a 30-day randomised controlled trial (RCT) involving supplementation with a probiotic formulation, comprising B. longum R0175 and L. helveticus R0052, and reported a reduction in the depression among probiotic group subjects. Although the subjects, the probiotic strains and supplementation periods differed, the present study also demonstrated favourable effects on anxiety and depression. In the present cohort, the baseline anxiety and depression scores were relatively low, and only slight reductions were observed after the intervention. However, it is noteworthy that additional benefits were observed in elderly subjects with a favourable mental state. The observed reduction in anxiety in the present placebo group may be attributable to the effects of resistance training (Strickland and Smith, 2014).

Numerous studies have demonstrated the body fat-reducing effects of Bifidobacterium supplementation. Kondo et al. (2010) reported that $B$. breve B-3 inhibited body weight gain and body fat accumulation in mice with high-fat dietinduced obesity. Supplementation of B. breve B-3 reportedly regulates gene expression involved in lipid metabolism and response to stress in the liver (Kondo et al., 2013). In addition, Minami et al. (2015) conducted a 12-week $\mathrm{RCT}$ of a test food containing $B$. breve B-3 on a cohort of obese men/women aged 40-69 years (BMI: 24-30 kg/ $\mathrm{m}^{2}$ ) and reported a significant decrease in the body fat percentage in the probiotic group. In the present study, body weight and BMI showed a significant decrease in the probiotic group at 12 weeks. It is noteworthy that the results were not attributable to malnutrition because no subject showed malnutrition at baseline, according to the dietary survey and blood biochemistry tests (Supplementary Table 1). There were 7 overweight (BMI $\left.>25 \mathrm{~kg} / \mathrm{m}^{2}\right)$ and 13 normal (BMI 18.5-25 kg/m²) participants at baseline in the probiotic group); no participant with inadequate body weight reduction $\left(\mathrm{BMI}<18.5 \mathrm{~kg} / \mathrm{m}^{2}\right)$ was observed during intervention period. The prevalence of overweight/obesity in the global geriatric population has increased (UN, 2015), and the results of the present study suggest that probiotics could contribute to gradual body weight loss or body weight control in the elderly. However, subjects with normal BMI were included in this study; therefore, the changes in the body weight and BMI were minor and there were no intergroup differences. This result needs to be confirmed in future studies on overweight elderly subjects.

The incidence of constipation increases with age (Thompson and Heaton, 1980), and this disorder affects the health-related QOL (Wald et al., 2007). The present study demonstrated a significant increase in the defecation frequency in the probiotic group. Self-recorded scores suggest that faecal properties remained normal, with no impact on the post-defecation comfort. Kondo et al. (2013) conducted a 16-week RCT of a test food containing $B$. longum BB536, also included in the present study, with a mixed-sex cohort of subjects aged 65-102 years on enteral nutrition. The probiotic intervention led to an increased defecation frequency in a subgroup with low baseline defecation frequency ( $\leq 4$ times a week) and a decrease in the defecation frequency of a subgroup with high baseline defecation frequency ( $\geq 10$ times a week), suggesting probiotic-related normalisation (Kondo et al., 2013). Furthermore, previous crossover interventional studies of B. longum BB536 in adult women have shown an increase in the defecation frequency at 2 weeks (Ogata et al., 1997; Yaeshima et al., 2001), and optimisation of the intestinal microbiota, enzyme activities related to the assimilation of ammonia, and content of water and acetic acids that could influence the intestinal environment (Ogata et al., 1997). Thus, the present results are consistent with those of previous Bifidobacterium studies and provide additional evidence of its propensity to maintain intestinal function.

\section{Limitations}

The present study had two main limitations. First, the statistical power was limited owing to the small sample size. Second, the intervention period was relatively short. The main strengths of the study were its RCT design and the optimal adherence rates $(\geq 99 \%)$ of the two study groups. Third, we did not exclude the participants who consumed dairy products including yogurt; this may have influenced their gut microbiota composition. Despite the limitations, to our knowledge, this is the first RCT to demonstrate the beneficial effects of combined bifidobacteria supplementation and moderate resistance training on several health-related parameters, such as body weight, bowel movement and mental health of healthy elderly subjects. Further studies are warranted to 
replicate the present findings and elucidate the impact of the bifidobacteria on geriatric cognitive function.

\section{Conclusions}

The present results suggest that, in healthy, elderly subjects, combined probiotic bifidobacteria supplementation and moderate resistance training may have beneficial effects on the cognitive function and other health-related parameters, such as body weight, bowel habits and mental state.

\section{Supplementary material}

Supplementary material can be found online at https://doi. org/10.3920/BM2017.0193.

Table S1. Blood test results.

\section{Acknowledgements}

We are grateful to the participants of the present study. We are grateful to Dr. Yuji Wada and Dr. Satoshi Suto for their valuable assistance in the development of the cognitive function programme and to Dr. Kazuhiko Kotani for logistic help throughout the study. This work was supported by Cabinet Office, Government of Japan, Cross-ministerial Strategic Innovation Promotion Program (SIP Project ID:14533567), 'Technologies for creating next-generation agriculture, forestry and fisheries' (funding agency: Biooriented Technology Research Advancement Institution, NARO).

\section{References}

AI Omran, Y. and Aziz, Q., 2014. The brain-gut axis in health and disease. Advances in Experimental Medicine and Biology 817: 135-153

Akbari, E., Asemi, Z., Daneshvar Kakfaki, R., Bahmani, F., Kouchaki, E., Tamtaji, O.R., Hamidi, G.A. and Salami, M., 2016. Effect of probiotic supplementation on cognitive function and metabolic status in Alzheimer's disease: a randomized, double-blind and controlled trial. Frontiers in Aging Neuroscience 8: 256.

Allen, A.P., Hutch, W., Borre, Y.E., Kennedy, P.J., Temko, A., Boylan, G., Murphy, E., Cryan, J.F., Dinan, T.G. and Clarke, G., 2016. Bifidobacterium longum 1714 as a translational psychobiotic: modulation of stress, electrophysiology and neurocognition in healthy volunteers. Translational Psychiatry 6: e939.

Barton, W., Penney, N.C., Cronin, O., Garcia-Perez, I., Molloy, M.G., Holmes, E., Shanahan, F., Cotter, P.D. and O'Sullivan, O., 2017. The microbiome of professional athletes differs from that of more sedentary subjects in composition and particularly at the functional metabolic level. Gut 67: 625-633.
Bercik, P., Park, A.J., Sinclair, D., Khoshdel, A., Lu, J., Huang, X., Deng, Y., Blennerhassett, P.A., Fahnestock, M., Moine, D., Berger, B., Huizinga, J.D., Kunze, W., McLean, P.G., Bergonzelli, G.E., Collins, S.M. and Verdu, E.F., 2011. The anxiolytic effect of Bifidobacterium longum NCC3001 involves vagal pathways for gut-brain communication. Neurogastroenterology and Motility 23: 1132-1139.

Bhatia, V. and Tandon, R.K., 2005. Stress and the gastrointestinal tract. Journal of Gastroenterology and Hepatology 20: 332-339.

Cerdá, B., Pérez, M., Pérez-Santiago, J.D., Tornero-Aguilera, J.F., González-Soltero, R. and Larrosa, M., 2016. Gut microbiota modification: another piece in the puzzle of the benefits of physical exercise in health? Frontiers in Physiology 7: 51.

Chung, Y.C., Jin, H.M., Cui, Y., Kim, D.S., Jung, J.M., Park, J.I., Jung, E.S., Choi. E.K. and Chae, S.W., 2014. Fermented milk of Lactobacillus helveticus IDCC3801 improves cognitive functioning during cognitive fatigue tests in healthy older adults. Journal of Functional Foods 10: 465-474.

Cryan, J.F. and Dinan, T.G., 2012. Mind-altering microorganisms: the impact of the gut microbiota on brain and behavior. Nature Reviews Neuroscience 13: 701-712.

Desbonnet, L., Garrett, L., Clarke, G., Kiely, B., Cryan, J.F. and Dinan, T.G., 2010. Effects of the probiotic Bifidobacterium infantis in the maternal separation model of depression. Neuroscience 170: 1179-1188.

Diaz Heijtz, R., Wang, S., Anuar, F., Qian, Y., Björkholm, B., Samuelsson, A., Hibberd, M.L., Forssberg, H. and Pettersson, S., 2011. Normal gut microbiota modulates brain development and behavior. Proceedings of the National Academy of Sciences of the USA 108: 3047-3052.

Faul, F., Erdfelder, E., Buchner, A. and Lang, A.G., 2009. Statistical power analyses using G*Power 3.1: tests for correlation and regression analyses. Behavior Research Methods 41: 1149-1160.

Fujiwara, Y., Suzuki, H., Yasunaga, M., Sugiyama, M., Ijuin, M., Sakuma, N., Inagaki, H., Iwasa, H., Ura, C., Yatomi, N., Ishii, K., Tokumaru, A.M., Homma, A., Nasreddine, Z. and Shinkai, S., 2010. Brief screening tool for mild cognitive impairment in older Japanese: validation of the Japanese version of the Montreal Cognitive Assessment. Geriatrics and Gerontology International 10: 225-232.

Fung, T.C., Olson, C.A. and Hsiao, E.Y., 2017. Interactions between the microbiota, immune and nervous systems in health and disease. Nature Neuroscience 20: 145-155.

Gallucci, M., Mazzuco, S., Ongaro, F., Di Giorgi, E., Mecocci, P., Cesari, M., Albani, D., Forloni, G.L., Durante, E., Gajo, G.B., Zanardo, A., Siculi, M., Caberlotto, L. and Regini, C., 2013. Body mass index, lifestyles, physical performance and cognitive decline: the 'Treviso Longeva (TRELONG)' study. Journal of Nutrition Health and Aging $17: 378-384$

Gareau, M.G., 2014. Microbiota-gut-brain axis and cognitive function. In: Lyte, M. and Cryan, J.F. (eds.) Microbial endocrinology: the microbiota-gut-brain axis in health and disease. Advances in Experimental Medicine and Biology, Springer, New York, NY, USA, pp. 357-368.

Gareau, M.G., Sherman, P.M. and Walker, W.A., 2010. Probiotics and the gut microbiota in intestinal health and disease. Nature Reviews Gastroenterology \& Hepatology 7: 503-514. 
Gustafson, D., Rothenberg, E., Blennow, K., Steen, B. and Skoog, I., 2003. An 18-year follow-up of overweight and risk of Alzheimer disease. Archives of Internal Medicine 163: 1524-1528.

Kamijo, K., Hayashi, Y., Sakai, T., Yahiro, T., Tanaka, K. and Nishihira, Y., 2009. Acute effects of aerobic exercise on cognitive function in older adults. Journals of Gerontology, series B: Psychological Sciences and Social Sciences 64: 356-363.

Kobayashi, Y., Sugahara, H., Shimada, K., Mitsuyama, E., Kuhara, T., Yasuoka, A., Kondo, T., Abe, K. and Xiao, J.Z., 2017. Therapeutic potential of Bifidobacterium breve strain A1 for preventing cognitive impairment in Alzheimer's disease. Scientific Reports 7: 13510.

Kondo, J., Xiao, J.Z., Shirahata, A., Baba, M., Abe, A., Ogawa, K. and Shimoda, T., 2013. Modulatory effects of Bifidobacterium longum BB536 on defecation in elderly patients receiving enteral feeding. World Journal of Gastroenterology 19: 2162-2170.

Kondo, S., Kamei, A., Xiao, J.Z., Iwatsuki, K. and Abe, K., 2013. Bifidobacterium breve B-3 exerts metabolic syndrome-suppressing effects in the liver of diet-induced obese mice: a DNA microarray analysis. Beneficial Microbes 4: 247-251.

Kondo, S., Xiao, J.Z., Satoh, T., Odamaki, T., Takahashi, S., Sugahara, H., Yaeshima, T., Iwatsuki, K., Kamei, A. and Abe, K., 2010. Antiobesity effects of Bifidobacterium breve strain B-3 supplementation in a mouse model with high-fat diet-induced obesity. Bioscience, Biotechnology and Biochemistry 74: 1656-1561.

Kroenke, K., Spitzer, R.L. and Williams, J.B., 2001. The PHQ-9: validity of a brief depression severity measure. Journal of General Internal Medicine 16: 606-613.

Lilamand, M., Kelaiditi, E., Cesari, M., Raynaud-Simon, A., Ghisolfi, A., Guyonnet, S., Vellas, B. and Van Kan, G.A., 2015. Validation of the mini nutritional assessment-short form in a population of frail elders without disability. analysis of the toulouse frailty platform population in 2013. Journal of Nutrition Health and Aging 19: 570-574.

Löwe, B., Decker, O., Müller, S., Brähler, E., Schellberg, D., Herzog, W. and Herzberg, P.Y., 2008. Validation and standardization of the Generalized Anxiety Disorder Screener (GAD-7) in the general population. Medical Care 46: 266-274.

Löwe, B., Wahl, I., Rose, M., Spitzer, C., Glaesmer, H., Wingenfeld, K., Schneider, A. and Brähler, E., 2010. A 4-item measure of depression and anxiety: validation and standardization of the Patient Health Questionnaire-4 (PHQ-4) in the general population. Journal of Affective Disorders 122: 86-95.

Matsumoto, M., Inoue, R., Tsukahara, T., Ushida, K., Chiji, H., Matsubara, N. and Hara, H., 2008. Voluntary running exercise alters microbiota composition and increases n-butyrate concentration in the rat cecum. Bioscience, Biotechnology, and Biochemistry 72: 572-576.

Messaoudi, M., Lalonde, R., Violle, N., Javelot, H., Desor, D., Nejdi, A., Bisson, J.F., Rougeot, C., Pichelin, M., Cazaubiel, M. and Cazaubiel, J.M., 2011. Assessment of psychotropic-like properties of a probiotic formulation (Lactobacillus helveticus R0052 and Bifidobacterium longum R0175) in rats and human subjects. British Journal of Nutrition 105: 755-764.
Minami, J., Kondo, S., Yanagisawa, N., Odamaki, T., Xiao, J.Z., Abe, F., Nakajima, S., Hamamoto, Y., Saitoh, S. and Shimoda, T., 2015. Oral administration of Bifidobacterium breve B-3 modifies metabolic functions in adults with obese tendencies in a randomized controlled trial. Journal of Nutritional Science 4: e17.

Mu, C., Yang, Y. and Zhu, W., 2016. Gut microbiota: the brain peacekeeper. Frontiers in Microbiology 7: 345.

Muramatsu, K., Miyaoka, H., Kamijima, K., Muramatsu, Y., Fuse, K., Yoshimine, F., Hosaka, M., Kutsumi, R., Majima, I., Katagiri, A., Murakami, S., Seino, H., Tanaka, H., Narita, K., Arakawa, M., Sakurai, K., Fujimura, T. and Baba, S., 2010. Validity and utility of a Japanese version of the GAD-7. Japanese Journal of Psychosomatic Medicine 50: 166.

Muramatsu, K., Miyaoka, H., Kamijima, K., Muramatsu, Y., Yoshida, M., Otsubo, T. and Gejyo, F., 2007. The patient health questionnaire, Japanese version: validity according to the miniinternational neuropsychiatric interview-plus. Psychological Reports 101: 952-960.

Nagai, N., Sakane, N., Kotani, K., Hamada, T., Tsuzaki, K. and Moritani, T., 2011. Uncoupling protein 1 gene $-3826 \mathrm{~A} / \mathrm{G}$ polymorphism is associated with weight loss on a short-term, controlled-energy diet in young women. Nutrition Research 31: 255-261.

Northey, J.M., Cherbuin, N., Pumpa, K.L., Smee, D.J. and Rattray, B., 2017. Exercise interventions for cognitive function in adults older than 50: a systematic review with meta-analysis. British Journal of Sports Medicine 52(3): 154-160.

O’Donnell, L.J., Virjee, J. and Heaton, K.W., 1990. Detection of pseudodiarrhoea by simple clinical assessment of intestinal transit rate. British Medical Journal 17: 300.

Ogata, T., Nakamura, T., Anjitsu, K., Yaeshima, T., Takahashi, S., Fukuwatari, Y., Ishibashi, N., Hayasawa, H., Fujisawa, T. and Iino, H., 1997. Effect of Bifidobacterium longum BB536 administration on the intestinal environment, defecation frequency and fecal characteristics of human volunteers. Bioscience Microflora 16: 53-58.

Portincasa, P., Maggipinto, A., Berardino, M., Bonfrate, L., Costin, S., Todarello, O., Palasciano, G., Wang, D.Q. and Dumitrascu, D.L., 2009. Assessing gastrointestinal symptoms and perception, quality of life, motility, and autonomic neuropathy in clinical studies. Journal of Gastrointestinal and Liver Diseases 18: 205-211.

Quigley, E.M.M., 2017. Microbiota-brain-gut axis and neurodegenerative diseases. Current Neurology and Neuroscience Reports 17: 94.

Ruiz, L., Delgado, S., Ruas-Madiedo, P., Sánchez, B. and Margolles, A., 2017. bifidobacteria and their molecular communication with the immune system. Frontiers in Microbiology 8: 2345.

Sampson, T.R. and Mazmanian, S.K., 2015. Control of brain development, function, and behavior by the microbiome. Cell Host and Microbe 17: 565-576.

Silverman, M.N. and Deuster, P.A., 2014. Biological mechanisms underlying the role of physical fitness in health and resilience. Interface Focus 4: 20140040.

Spitzer, R.L., Kroenke, K., Williams, J.B. and Löwe, B., 2006. A brief measure for assessing generalized anxiety disorder: the GAD-7. Archives of Internal Medicine 166: 1092-1097. 
Strickland, J.C. and Smith, M.A., 2014. The anxiolytic effects of resistance exercise. Frontiers in Psychology 5: 753.

Sudo, N., Chida, Y., Aiba, Y., Sonoda, J, Oyama, N., Yu, X.N., Kubo, C. and Koga, Y., 2004. Postnatal microbial colonization programs the hypothalamic-pituitary-adrenal system for stress response in mice. Journal of Physiology 558: 263-275.

Thompson, W.G. and Heaton, K.W., 1980. Functional bowel disorders in apparently healthy people. Gastroenterology 79: 283-288.

Ticinesi, A., Lauretani, F., Milani, C., Nouvenne, A., Tana, C., Del Rio, D., Maggio, M., Ventura, M. and Meschi, T., 2017. Aging gut microbiota at the cross-road between nutrition, physical frailty, and sarcopenia: is there a gut-muscle axis? Nutrients 9: e1303.

United Nations, 2015. World population ageing. Department of Economic and Social Affairs Population Division. United Nations, New York, NY, USA, pp. 16-19. https://tinyurl.com/jtscjoa.

Vaughan, S., Wallis, M., Polit, D., Steele, M., Shum, D. and Morris, N., 2014. The effects of multimodal exercise on cognitive and physical functioning and brain-derived neurotrophic factor in older women: a randomized controlled trial. Age and Ageing 43: 623-629.

Wald, A., Scarpignato, C., Kamm, M.A., Mueller-Lissner, S., Helfrich, I., Schuijt, C., Bubeck, J., Limoni, C. and Petrini, O., 2007. The burden of constipation on quality of life: results of a multinational survey. Alimentary Pharmacology and Therapeutics 26: 227-236.
Walsh, J.N., Manor, B., Hausdorff, J., Novak, V., Lipsitz, L., Gow, B., Macklin, E.A., Peng, C.K. and Wayne, P.M., 2015. Impact of shortand long-term Tai chi mind-body exercise training on cognitive function in healthy adults: results from a hybrid observational study and randomized trial. Global Advances in Health and Medicine 4: 38-48

Wang, Z.H., Gao, Q.Y. and Fang, J.Y., 2013, Meta-analysis of the efficacy and safety of Lactobacillus-containing and Bifidobacteriumcontaining probiotic compound preparation in Helicobacter pylori eradication therapy. Journal of Clinical Gastroenterology 47: 25-32.

Xu, F., Delmonico, M.J., Lofgren, I.E., Uy, K.M., Maris, S.A., Quintanilla, D., Taetzsch, A.G., Letendre, J. and Mahler, L., 2017. Effect of a combined Tai chi, resistance training and dietary intervention on cognitive function in obese older women. Journal of Frailty and Aging 6: 167-171.

Yaeshima, T., Takahashi, S., Ogura, A., Konno, T., Iwatsuki, K., Ishibashi, N. and Hayasawa, H., 2001. Effect of non-fermented milk containing Bifidobacterium longum BB536 on the defecation frequency and fecal characteristics in healthy adults. Journal of Nutritional Food 4: 1-6. 
